\title{
Effects of random discrete impurities in ultra-short MOSFET using 3D Monte Carlo simulation
}

\author{
Sylvain Barraud ${ }^{(*)}$, Philippe Dollfus, Sylvie Galdin, Patrice Hesto \\ IEF, CNRS UMR 8622, Bâtiment 220, Université Paris-Sud, 91405 Orsay, France \\ (*) present address: LETI/CEA, 17 rue des Martyrs, 38054 Grenoble cedex 9, France \\ philippe.dollfus@ief.u-psud.fr
}

\begin{abstract}
This paper presents theoretical investigation of electron transport in the channel of $50 \mathrm{~nm}$ MOSFET with discrete random dopant distribution. The work is based on three-dimensional device Monte Carlo simulation. A model of electron-ion interaction suitable for discrete dopants has been developed by carefully separating the long-range and short-range parts of Coulomb potential effects. This model is validated through mobility calculation in silicon resistors and applied to MOSFET simulation. Discrete dopant distributions are compared with the case of continuous doping in terms of drain current. The transport is analysed through profile and cartography of electron concentration, electron velocity and electron flux. The presence of impurity in the inversion layer is shown to strongly affect locally these quantities and then the drain current.
\end{abstract}

\section{Introduction}

With the downscaling of MOSFETs to sub-100 nm lateral dimensions many physical and technological features pose in new terms the design of next CMOS generations. Among the new important issues the inescapable statistical fluctuations in the number and position of doping impurities yield spreading in device characteristics as the threshold voltage and the off-state current, to such a point that it may be unacceptable to CMOS operation [1,2]. These random dopant fluctuations should be an important feature also in the theoretical treatment of carrier transport in aggressively scaled-down devices. This paper aims at addressing theoretical aspects of this issue through 3D device Monte Carlo simulation.

In the past years, the effects of discrete dopant random distribution in MOSFET channel have been investigated using analytical [3] or Drift-Diffusion (DD) approaches [4]. 3D 'atomistic' simulators have also been developed specifically for studying threshold voltage fluctuations [5]. The DD method is very useful because of its simplicity and fast computing times, which allows statistical investigation on a wide of dopant random distributions. However, 3D Monte Carlo (MC) simulation should provide a more realistic transport description in ultra-short MOSFET. It is the motivation of this paper. The model used to describe electron-ion (e-i) interaction in the frame of 3D MC simulation with discrete impurities is briefly summarised. It is based on the long-range Particle-Mesh approach, i.e. on the solution of Poisson's equation. A correction is applied in the short-range domain to remove the Coulomb potential errors inherent in the Particle-Mesh method. This model is then used to study the transport in $50 \mathrm{~nm}$ gate length MOSFETs with a channel impurity random distribution determined from Monte Carlo simulation of the implantation steps.

\section{Electron-impurity scattering model}

In conventional simulation models with continuous doping concentrations the electron-impurity interaction is considered as a perturbation characterised by a scattering rate. If ionised-impurities and carriers are treated simultaneously as point-charge particles, the electronimpurity interaction is to be considered in the carrier motion calculation directly. Among the particle simulation methods initially developed by Hockney [6] the Particle-Particle (PP) model and the Particle-Mesh (PM) model have to be mentioned. The first consists in calculating the Coulomb force applied to a particle by summing the contributions of all other particles. Less computational demanding is the PM model that considers the local electric field acting on a particle as the gradient of the potential distribution resulting from the solution of Poisson's equation in a pre-defined meshing. It is the socalled long-range approach of the interaction. We have adopted this method by modifying the conventional 3D Monte Carlo simulator previously developed for continuous doping $[7,8]$.

To include the e-i long-range interaction with discrete impurities the change in the MC algorithm essentially consists in removing the short-range electron-impurity scattering mechanism from the usual set of possible scattering events while taking into account the actual position of impurities to solve 3D Poisson's equation. According to its position, each impurity charge is 
assigned to a cell. The 3D Poisson's equation is solved using a finite-element method in a non-uniform rectilinear grid. In principle, the so-called mesh force derived from this self-consistent potential should fully include the e-i interaction through a long-range approach. However, once the grid has been defined, the particle charges have to be assigned to the grid nodes before solving Poisson's equation and we no longer can consider particles as point particles. This is the origin of the main limitation of this technique that is to be corrected in the short-range domain $[9,10]$. Indeed, at very short distance from the impurity the mesh force experienced by an electron approaches zero due to the mesh-induced charge smoothing, while the real Coulomb force tends to infinity. The validity of the PM approach is thus limited by inadequacies between the concepts of point charge and spatial discretization. It has been demonstrated indeed that the discrimination between the long-range and short-range parts of the Coulomb potential is essential [11]. A correction to the mesh force used in the PM method is thus necessary in the vicinity of impurities, i.e. in the cells where the mesh force calculation is dubious.

An approach based on PM/PP coupling has been developed to correct the Coulomb force near each impurity $[9,10]$. This $\mathrm{P}^{3} \mathrm{M}$ algorithm consists of a direct calculation of short-range forces between particles in predefined short-range regions using the PP technique. We have proposed an alternative MC approach to correctly treat the carrier-impurity interaction in the short-range domain using a scattering process [12]. This correction technique used in our model is briefly summarised now.

In the vicinity of each impurity a short-range zone (SRZ) is defined. Inside a SRZ the field applied to electrons is deduced from the potential at the zone boundaries resulting from Poisson's equation solution. The mesh force thus vanishes and the electron-impurity interaction is described by a short-range scattering mechanism integrated into the MC scattering procedure, similarly to the standard method used for continuous doping. Outside the SRZ, the long-range mesh force is applied without any impurity scattering, as in the pure PM method. This new model is hereafter called Corrected Particle-Mesh (CPM) model.

One of the main feature of the model is the correct definition of SRZ space extension. This question is discussed in details in Ref. 12. It emerges from this discussion that (i) the mesh size $\Delta \mathrm{x}$ must be less than one tenth of the average distance between two impurities to correctly describe the long-range interaction, and (ii) a SRZ must be extended to the cube of 125 cells surrounding the impurity to correctly treat the shortrange scattering with unscreened Coulomb potential.

\section{Mobility calculation}

To validate this CPM model we have computed the low-field electron drift mobility in $\mathrm{Si}$ as a function of the doping concentration for comparison with experimental mobilities. All details about the band structure and the electron-phonon scattering parameters used in our model may be found in Ref. 8. The simulated structures are 3D silicon resistors with two terminal contacts to which the potential is fixed so as to induce an average driving field of $0.5 \mathrm{kV} / \mathrm{cm}$. Periodic boundary conditions are used, i.e. each carrier leaving the device through a contact is reinjected at the other side with the same wave vector. It allows recovering bulk transport conditions without simulating very long structures. According to the doping concentration $N_{D}$, the size of resistors is adjusted to have the same number of donor atoms $N_{a t}=45$ in all structures.

The mobilities calculated with the CPM model are plotted in Fig. 3 (closed circles). The solid line shows the fit of experimental mobilities [13] for comparison. The model matches very well with the experiments. Although the model does not take into account the degeneracy effects, the agreement is satisfying also at very high doping concentration, up to $6.4 \times 10^{19} \mathrm{~cm}^{-3}$.

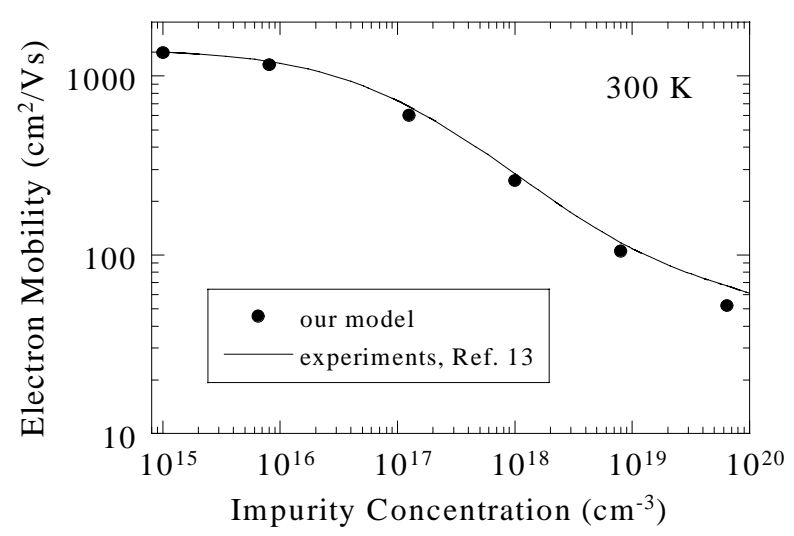

Figure 1. Electron mobility as a function of donor concentration. The solid line represents the fitting curve of experimental data (Ref. 13).

\section{MOSFET simulation}

This model is now applied to the study of a MOSFET with discrete channel impurities. The channel length and width are $50 \mathrm{~nm}$, the gate oxide thickness is $1 \mathrm{~nm}$, and the junction depth is $15 \mathrm{~nm}$. The dopant implantation steps have been simulated using a Monte Carlo model [14]. In the active channel region the resulting discrete distribution of boron atoms is considered for the device simulation with an average concentration of $10^{18} \mathrm{~cm}^{-3}$. In the other parts of the device, i.e. substrate and source/drain regions, smooth doping profiles are used. For the purpose of comparison a similar MOSFET has been simulated with a uniform and continuous doping concentration in the channel. 
The $\mathrm{I}_{\mathrm{D}}-\mathrm{V}_{\mathrm{GS}}$ characteristics at $\mathrm{V}_{\mathrm{DS}}=0.6 \mathrm{~V}$ are plotted in Fig. 2 for the continuous doping (solid line) and for the discrete doping (dashed line). For this specific dopant distribution (called "discrete-1" in the figure) the current is significantly higher than in the continuous case. In Fig. 3 is plotted the cartography of longitudinal electric field just under the gate oxide for "discrete-1" distribution. This view makes clearly visible three impurities present in the inversion layers with corresponding cubic SRZ in which the mesh force vanishes. Repulsive electric field appears on the sides of SRZ. To analyse the transport in inversion layer we define two typical lines between source and drain, as represented in Fig. 3 : an "impurity" line (going through one impurity cell localized at $X=49.5 \mathrm{~nm}$ ) and a "noimpurity" line.

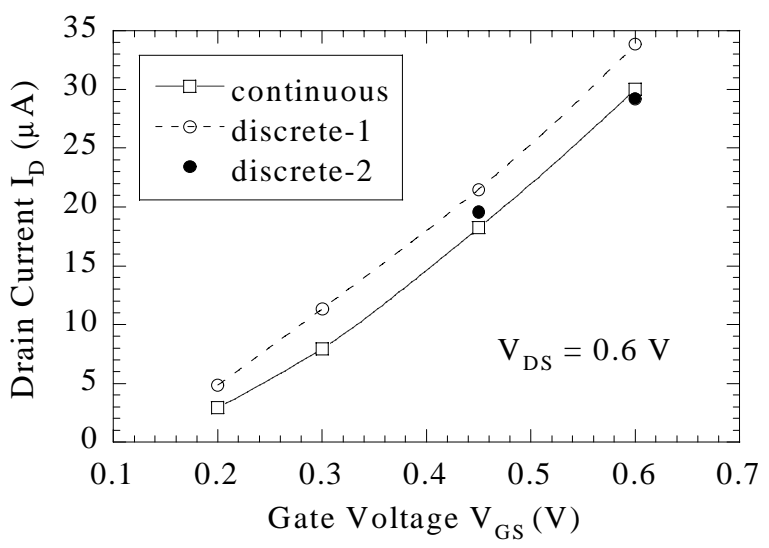

Figure 2. $I_{D}-V_{G S}$ curves of simulated MOSFETs with continuous and discrete channel doping. "discrete-1" and "discrete-2" correspond to two different dopant implantations.

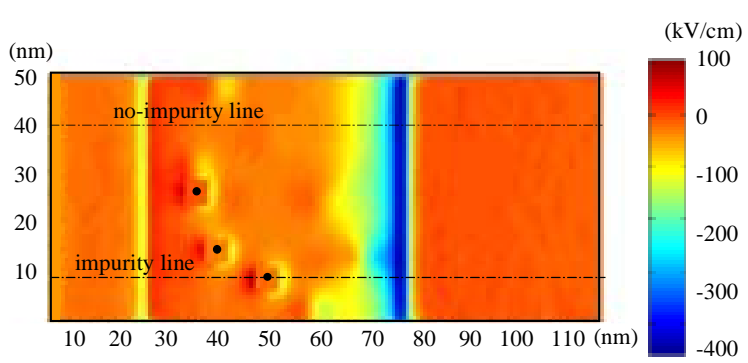

Figure 3. Longitudinal electric field cartography (top view) in the inversion layer between source and drain for "discrete-1" dopant distribution with three impurities $\left(\mathrm{V}_{\mathrm{GS}}=0.45 \mathrm{~V}, \mathrm{~V}_{\mathrm{DS}}=0.6 \mathrm{~V}\right)$

Have a look to the electron density and velocity profiles plotted in Figs. 5 and 6 (for $\mathrm{V}_{\mathrm{GS}}=0.45 \mathrm{~V}$, $\mathrm{V}_{\mathrm{DS}}=0.6 \mathrm{~V}$ ). These profiles are drawn between source an drain along three lines : both "impurity" (dashed) and "no-impurity" line (dotted), together with a line corresponding to the continuous doping (solid). When compared to the continuous case the density is weakly modified along the no-impurity line but strongly affected along the impurity line, specially around the SRZ. The electrons seriously slow down near the SRZ but they are significantly accelerated on a large part of the no-impurity line. The largest part of the current circulate in the regions free of impurity where the velocity is higher than in the continuous doping case. Hence, the current shift depends on the number, and possibly the position of impurity atoms in the inversion layer assumed to be about $3 \mathrm{~nm}$ thick. This number is 3 in this case ("discrete-1" in Fig. 2) but it is 7 for another implantation process ("discrete-2" in Fig. 2) which reduces the current to a value close to the continuous case.

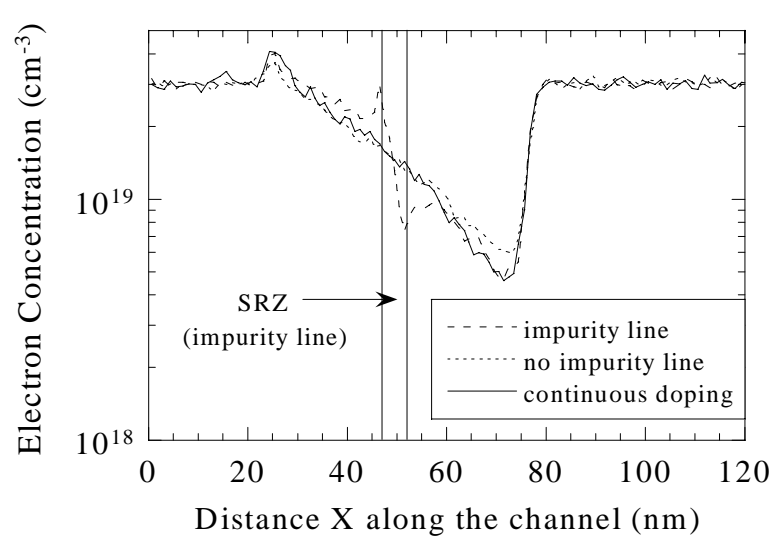

Figure 4. Electron density along three lines in the source-drain direction for continuous and discrete channel doping $\left(\mathrm{V}_{\mathrm{GS}}=0.45 \mathrm{~V}, \mathrm{~V}_{\mathrm{DS}}=0.6 \mathrm{~V}\right)$.

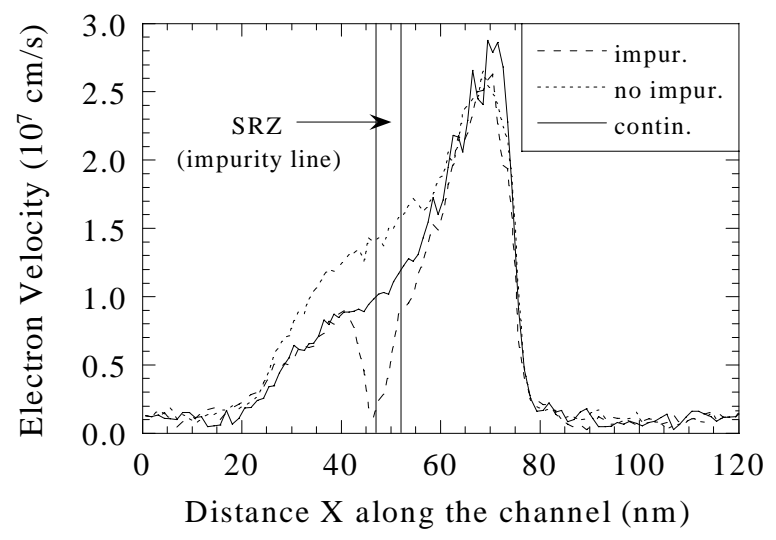

Figure 5. Electron velocity along three lines in the source-drain direction for continuous and discrete channel doping $\left(\mathrm{V}_{\mathrm{GS}}=0.45 \mathrm{~V}, \mathrm{~V}_{\mathrm{DS}}=0.6 \mathrm{~V}\right)$.

In Fig. 6 is plotted the cartography of electron flux between source and drain for both dopant distributions. It clearly shows the low-flux regions around impurities (blue regions) and the high-flux regions between them (red). Due to higher amount of impurities in the inversion layer the high-flux region for "discrete-2" distribution (seven impurities) is narrower than for "discrete-1" distribution (three impurities). It explains the lower current observed in Fig. 2. 


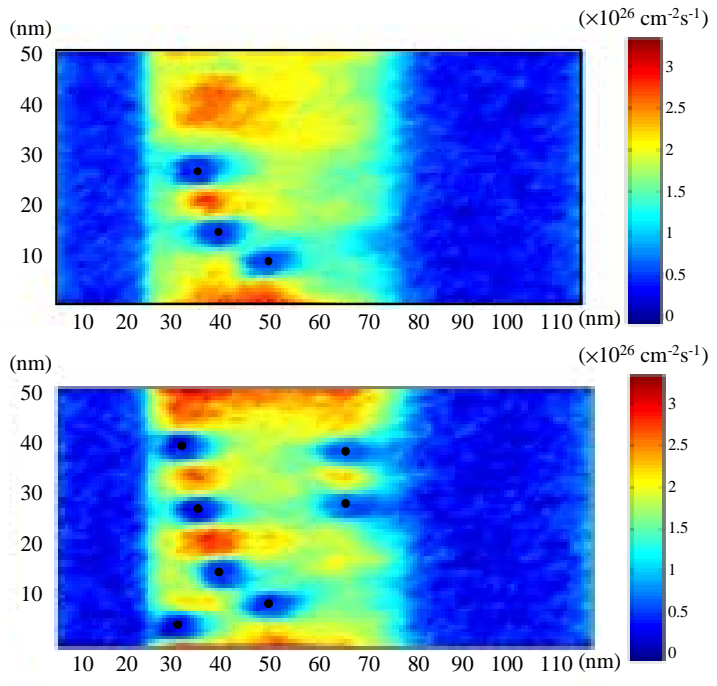

Figure 6. Cartography of electron flux under the gate oxide between source and drain; (top) discrete-1 distribution; (bottom) discrete-2 distribution; $\left(\mathrm{V}_{\mathrm{GS}}=0.45 \mathrm{~V}, \mathrm{~V}_{\mathrm{DS}}=0.6 \mathrm{~V}\right)$

\section{Conclusion}

The standard 3D Monte Carlo algorithm has been modified to study the effects of discrete random dopant distribution on electrical parameters of sub-100 nm MOSFET. This approach allows to combining random dopant and non-stationary transport effects. The new electron-impurity interaction model is based on the discrimination between between the long-range and short-range parts of the Coulomb potential. It has been applied to 50-nm MOSFETs study with discrete impurity distribution. The presence of an impurity in the inversion layer is shown to strongly affect the local electron velocity. The current and threshold voltage shifts between devices may be related to the number and position of such impurities.

\section{References}

[1] T. Mizuno, "Experimental study of threshold voltage fluctuations due to statistical variation of channel dopant number in MOSFET's", IEEE Trans. Electron Devices 41 (1994) 2216

[2] M.J.M. Pelgrom and M. Vertregt, "CMOS technology for mixed signal ICs" Solid-State Electron. 41 (1997) 967

[3] P.A. Stolk, F.P. Widdershoven and D.B.M. Klaasen, "Modeling statistical dopant fluctuations in MOS transistors" IEEE Trans. Electron Devices 45 (1998) 1960

[4] H.S. Wong and Y. Taur, "Trhee-dimensional atomistic simulation of discrete random dopant distribution effects in sub-0.1 $\mu \mathrm{m} "$ IEDM'93 Tech. Dig. (1993) 705

[5] A. Asenov, "Random dopant induced threshold voltage lowering and fluctuations in sub- $0.1 \mu \mathrm{m}$
MOSFET" IEEE Trans. Electron Devices 45 (1998) 2505

[6] R. W. Hockney and J. W. Eastwood, in Computer Simulation Using Particles, (McGraw-Hill, New York, 1981)

[7] C. Brisset, P. Dollfus, O. Musseau, J.-L. Leray, P. Hesto, "Theoretical study of SEUs in $0.25-\mu \mathrm{m}$ fullydepleted CMOS/SOI technology" IEEE Trans. Nucl. Sci. 41 (1994) 2297

[8] P. Dollfus, "Si/ $\mathrm{Si}_{1-\mathrm{x}} \mathrm{Ge}_{\mathrm{x}}$ heterostructures: electron transport and field-effect transistor operation using Monte Carlo simulation" J. Appl. Phys. 82 (1997) 3911

[9] W.J. Gross, D. Vasileska and D.K. Ferry, "A novel approach for introducing the electron-electron and electron-impurity interactions in particle-based simulations" IEEE Electron Device Lett. 20 (1999) 463

[10] C.J. Wordelman and U. Ravaioli, "Integration of a particle-particle-particle-mesh algorithm with the ensemble Monte Carlo method for the simulation of ultra-small semiconductor devices" IEEE Trans. Electron Devices 47 (2000), p. 410

[11] N. Sano, K. Matsuzawa, M. Mukai, and N. Nakayama, "Long-range and short range coulomb potentials in threshold characteristics under discrete dopants in sub-0.1 $\mu \mathrm{m}$ Si-MOSFETs" IEDM'2000 Tech. Dig. (2000) 275

[12] S. Barraud, P. Dollfus, S. Galdin, P. Hesto, "Short-range and long-range Coulomb interactions for 3D Monte Carlo device simulation with discrete impurity distribution ", Solid-State Electron., in press

[13] G. Masetti, M. Severi, and S. Solmi, "Modeling of carrier mobility against carrier concentration in arsenic-, phosphorus-, and boron-doped silicon" IEEE Trans. Electron Devices 30 (1983) 764

[14] L. Rajaonarison, P. Hesto, J.-F. Pône and P. Dollfus, "Monte-Carlo simulation of submicron devices and processes" Proc. SISDEP'91 (Hartung-Gorre Verlag, Germany 1991) p. 513 